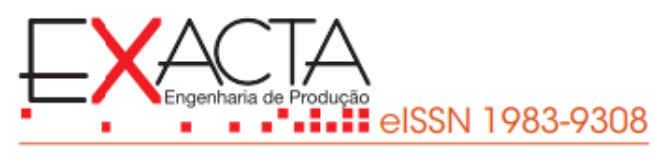

\title{
TOMADA DE DECISÃO EM SISTEMAS CIBER-FÍSICOS: UMA ANÁLISE BIBLIOMÉTRICA
}

\author{
DECISION-MAKING IN CYBER-PHYSICAL SYSTEMS: \\ A BIBLIOMETRIC ANALYSIS
}

Recebido em: 03 mar. 2019

Aprovado em: 27 jul. 2020

Versão do autor aceita publicada online: 27 jul. 2020

Publicado online: 12 maio 2021

Como citar esse artigo - American Psychological Association (APA):

Santana, H. L. \& Assumpção, M. R. P. (2023, jan./mar.). Tomada de decisão em sistemas ciberfísicos: uma análise bibliométrica. Exacta, 21(1), 1-21.

https://doi.org/10.5585/exactaep.2021.13163

Submeta seu artigo para este periódico 을

Dados Crossmark 



\title{
○ TOMADA DE DECISÃO EM SISTEMAS CIBER-FÍSICOS: UMA ANÁLISE BIBLIOMÉTRICA
}

\author{
DECISION-MAKING IN CYBER-PHYSICAL SYSTEMS: \\ A BIBLIOMETRIC ANALYSIS
}

\section{(D) Henrique Lima Santana ${ }^{1}$ \\ Maria Rita Pontes Assumpção²}

\begin{abstract}
${ }^{1}$ Universidade Metodista de Piracicaba
${ }^{2}$ Universidade Metodista de Piracicaba
\end{abstract}

Resumo: As mudanças no perfil de consumo por maior variedade de produtos têm elevado o nível de customização da produção. Para atender esta necessidade, a manufatura tem desenvolvido iniciativas que se consolidam na Indústria 4.0, caracterizada pelo uso de soluções para virtualização da produção, internet of things, big data, e em especial, pela utilização de sistemas ciber-físico (CPS). Isto significa reavaliar os inputs adotados na tomada de decisão. Diante desta problemática, coloca-se a questão que norteou a realização deste trabalho: como a tomada de decisão tem sido tratada no ambiente ciber-físico? Foi utilizado como método de pesquisa uma revisão da literatura em conjunto com análise bibliométrica. Os resultados indicam que os sistemas CPS necessitam de autonomia na decisão por intermédio de informações em tempo real de elementos da cadeia produtiva mais remotamente possível.

Palavras-chave: Industria 4.0. Produção inteligente. Decisão autônoma. Customização em massa. Previsão de demanda.

Abstract: The changes in the consumption profile for a greater variety of products have raised the production customization level. To meet this need, manufacturing has developed initiatives that are consolidated in Industry 4.0, characterized by the use of solutions for virtualization or digital twins, internet of things, big data, and in particular, the use of cyber-physical systems (CPS). This means re-evaluating the inputs adopted in the decision-making. Faced with this issue, the question that guided of this work arises: how has decision-making been treated in the cyberphysical environment? A literature review was used as a research method in conjunction with bibliometric analysis. The results indicate that CPS systems need decision autonomy through real-time information from elements of the production chain as remotely as possible.

Keywords: Industry 4.0. Smart production. Autonomous decision. Mass customization. Demand forecast. 
1 Introdução

Com a modificação das características de consumo, os produtos passaram a ter alta variação, flutuação da demanda e redução do ciclo de vida, impactando diretamente os sistemas regulares de produção. Os processos produtivos devem estar conectados por intermédio de uma robusta rede de informações para auxiliar a decisão (Bauer, Schoonmann, \& Reinhart, 2017).

Desta forma, o desafio da manufatura é aumentar a variedade dos produtos, por meio de decisões envoltas no ambiente de incertezas, sem afetar sua objetividade (Bai, 2009).

Com o objetivo de administrar estas circunstâncias, os sistemas ciber-físicos (CPS - CyberPhysical Systems) permitem interação entre organizações, dispositivos e operadores. Neste ambiente, o mundo físico é entrelaçado com sensores, atuadores e elementos computacionais que contribuem na tomada de decisão (Camarinha-Matos \& Afsarmanesh, 2014).

Exemplos dos dispositivos tecnológicos envolvidos com o sistema CPS que contribuem para a tomada de decisão são a internet das coisas (IoT - Internet of Things), sensoriamento, armazenamento na nuvem, produção inteligente, virtualização da produção (Vaidya, Ambad, \& Bhosle, 2018).

Esta variedade de tecnologia gera enormes quantidades de dados (big data) depositados em sistemas conectados na nuvem (armazenamento remoto), demandando o desenvolvimento de algoritmos capacitados no processamento para suportar a tomada da decisão (Papa, Zurutuza, \& Uribeetxeberria, 2016).

Mediante estas inovações, é necessário avaliar os fatores intervenientes na tomada de decisão que afetam a execução dos sistemas ciber-físicos e seus periféricos. Desta forma, é significativo destacar algumas questões a seguir.

É necessário considerar as informações históricas na configuração dos controladores de decisão de processo, visando proporcionar confiabilidade (Ilic, Xie, Khan, \& Moura, 2010).

Além dos históricos, de acordo com Swain, Couzin e Leonard (2012), as informações em tempo real são significativas na tomada de decisão em sistemas CPS, e assim, torna-se relevante a introdução de soluções que proporcionem comunicação de dados instantânea e atualização das informações.

A posse imediata da informação não é suficiente, é necessário integrar a cadeia produtiva para reduzir os impactos oriundos da variação da demanda no planejamento. Desta maneira, os sistemas CPS devem estender a abrangência de sua avaliação e considerar a influência dos elementos mais externos da cadeia na tomada de decisão (Tham \& Luo, 2013).

Contudo, a programação produtiva é alterada circunstancialmente. Zhao, Suryanarayanan e Simoes (2013) propõem a introdução do feedback como forma de comunicação para atualizar as condições de momento entre os componentes do CPS, e assim tornar a decisão mais assertiva. 
Com isso, a tomada de decisão nas organizações se tornou um processo estratégico que necessita se basear na gestão do conhecimento. Visando eliminar os aspectos intuitivos, a tomada de decisão tem sido orientada por uma vasta rede tecnológica disponível na indústria (Pereira, Ribeiro, \& Bianchini, 2014).

Essa tecnologia, a exemplo da virtualização da produção, internet of things e big data, é o suporte para introdução da decisão autônoma na indústria (Babiceanu \& Seker, 2016)

Para Wang, Torngren e Onori (2015) os sistemas ciber-físicos são depedentes dessa tomada de decisão autônoma, demandando um nível elevado de conectividade e a introduçao de controladores descentralizados.

Por fim, de acordo com conceitos do CPS, os módulos produtivos são autossuficientes, e geralmente desenvolvidos com softwares baseados em arquitetura de programação diversificada. A integração destes equipamentos deve garantir que as decisões autônomas proporcionem acuracidade e confiabilidade (Leitão, Colombo, \& Karnouskos, 2016).

Diante destas variáveis, o objetivo do trabalho é alcançar resposta para a seguinte questão: como a tomada de decisão tem sido tratada no ambiente ciber-físico? Desta maneira, é desenvolvida uma análise bibliométrica para buscar perspectiva relevante da produção científica no período de 2008 a 2018 sobre o tema - tomada de decisão no ambiente ciber-físico, considerando a aplicação de procedimentos para execução da pesquisa, análises das publicações, citações e referências, avaliação dos resultados e apresentação de uma análise conclusiva. Devido a especificidade das informações e para contribuir com encadeamento da leitura do artigo, os autores incluíram as figuras, quadros e tabelas como anexo, posteriormente as referências bibliográficas.

\section{Metodologia de pesquisa}

O termo bibliometria foi utilizado pela primeira vez, até onde pode ser apurado, no Journal of Documentation em dezembro de 1969. Naquele momento, R. A. Fairthorne escreveu um artigo denominado "descrição bibliométrica" sobre distribuição estatística aplicada ao campo da bibliometria (Broadus, 1987).

Para Mcburney e Novak (2002) o campo da bibliometria estuda padrões de publicação usando análises quantitativa e estatística. Segundo (Prasad \& Tata, 2005), a bibliometria busca identificar padrões nas publicações cientificas de forma apresentar tópicos relacionais de interesse.

Estudos bibliométricos são realizados em diferentes áreas do conhecimento, em diferentes línguas e sociedades. Uma das suas vantagens está no fato de analisar critérios de autoria, uso de palavras, classificação de periódicos, utilização de referências e mensuração da qualidade da produção (De Matos, Niyama, Neto, \& Marques, 2012). 
Segundo Henriques e Miguel (2015), com a evolução dos sistemas de informação, a utilização de dados bibliográficos procura facilitar o encontro de referências relevantes. Este processo pode ser desenvolvido de acordo com os seguintes procedimentos:

a) Seleção do banco de artigos brutos: composto pela definição das palavras-chave, definição bancos de dados, busca de artigos nos bancos de dados com as palavraschave;

b) Filtragem: composta pela filtragem dos artigos brutos quanto à redundância e duplicidade;

c) Filtragem quanto ao alinhamento do artigo integral: composto pela leitura dos artigos.

Com base nestas informações, este estudo apresenta uma análise bibliométrica visando respostas sobre a questão de pesquisa. Para realizar esta análise, são desenvolvidos procedimentos que contém uma amostragem inicial dos dados, onde são definidos o período da pesquisa, a escolha das bases de dados e os seletores de pesquisa. Posteriormente, é realizada a seleção dos artigos quanto a tipologia, área temática, cronologia, duplicidade e leitura do artigo para identificação da amostragem final. E por fim, realizada a análise bibliométrica dos artigos amostrados quanto as publicações, citações e referências.

\section{Procedimentos para seleção do referencial teórico}

A pesquisa deve utilizar referencial teórico que contribua na transformação de recursos em produtos e serviços para as áreas e funções de uma organização (Karlsson, 2008).

Para atingir este objetivo, a análise bibliométrica utiliza procedimentos que contribuem na produção científica por meio de indicadores (Gutiérrez-Salcedo, Martínez, Moral-Munoz, HerreraViedma, \& Cobo, 2018).

a) Indicadores de produção: número total de artigos publicados em um período de tempo, destacando a contagem de diferentes autores científicos;

b) Indicadores de impacto baseados em citações recebidas: total número de citações e número de citações por ano;

c) Indicadores baseados no impacto: indicação do fator de impacto do periódico, entre outros. 
A partir destas referências, são desenvolvidos procedimentos para compor a análise bibliométrica fundamentada no referencial teórico selecionado. Estes procedimentos são planejados em três etapas, detalhados em:
a) Amostragem inicial;
b) Seleção dos artigos para a pesquisa;
c) Análise bibliométrica da amostra de artigos.

\subsection{Amostragem inicial}

O uso da amostragem é uma prática para coletar informações e contribuir na análise da população dos registros. Na bibliomtria é determinante para atingir os objetivos da análise (Williams \& Bornmann, 2016).

\subsubsection{Período de coleta das informações}

As etapas de coleta de dados são realizadas em dezembro de 2018.

\subsubsection{Base de dados}

As bases utilizadas para pesquisar as amostras iniciais são a ScienceDirect, Scopus e Web of Science devido estas fontes terem relacionamento com o domínio da engenharia de produção (Wang \& Sun, 2018; Osorio \& Otieno, 2008). São selecionados e importados dados, a exemplo, o fator de impacto $J C R$ - Journal Citation Report.

\subsubsection{Seletores de pesquisa}

A pesquisa é realizada com as strings e operadores booleanos (("decision-making" AND "cyberphysical") OR ("decision-making" AND "industry 4.0")) a qual retornou com 2.193 trabalhos para composição da amostra, com representatividade em cada base de ScienceDirect (56\%), Scopus (31\%) e Web of Science (13\%).

\subsection{Seleção dos artigos para pesquisa}

Alinhados a amostragem inicial, são aplicados filtros visando encontrar trabalhos com atributos mais aderentes ao objetivo do artigo:

a) Tipologia, considerando apenas artigos revisados por pares devido a disponibilidade dos seus metadados, resulta no montante de 1.415 trabalhos; 
b) Área temática, considerando industrial engineering ou manufacturing engineering, devido a afinidade com o tema pesquisado, é selecionada uma nova amostra com 325 artigos;

c) Cronológico, considerando o período entre 2008 e 2018 devido a avaliação da modernidade sobre o tema. Não houve alteração da quantidade, indicando que os 325 artigos são contemporâneos ao período avaliado;

d) Duplicidade, considerando a origem em todas bases de pesquisa são descartados os artigos similares, reduzindo para 295 artigos amostrais.

Depois da leitura dos títulos e resumos dos artigos selecionados, são excluídos 50 trabalhos por não terem temática associada ao foco desta pesquisa. Portanto, a quantidade final de artigos da amostra é 245.

\subsection{Análise bibliométrica da amostra de artigos}

Uma vez definida a amostra, são importados os metadados disponíveis dos 245 artigos, tais como resumo, autores, palavras-chave, periódico, referências, número de citações, entre outros. Estes dados foram utilizados como entrada no software Microsoft Excel (Microsoft, 2016) permitindo tratar os arquivos texto e no software VOSviewer (Eck \& Waltman, 2018) para desenvolvimento da análise dos clusters (Lopes \& De Carvalho, 2012). Pesquisas também são realizadas no Google Scholar (Google LLC, 2018) para avaliação das citações e origem dos artigos.

\subsubsection{Análise das publicações}

A análise inicial é a cronologia de publicação dos artigos, possibilitando identificar os periódicos que mais publicaram, o percentual de participação e a comparação dos periódicos por meio do fator de impacto. Posteriormente, é possível realizar avaliação temporal quantitativa das publicações e a análise do país de procedência vinculado ao autor. Em seguida, são avaliadas as palavras-chaves dos artigos de acordo com a ocorrência e repetitividade, visando identificar o relacionamento com esta pesquisa. E de forma complementar, é apresentada análise dos clusters das palavras-chaves.

A tabela 1 (anexo I) apresenta o relacionamento entre os periódicos e o ano de publicação. Os 245 artigos são publicados em 73 periódicos demostrando o amplo nível interesse do tema para as áreas de industrial engineering ou manufacturing engineering.

Cinco periódicos representam cerca de 62\% das publicações (destaque em cinza na tabela 1) sendo eles: Procedia Manufacturing (27\%) com fator de impacto 0,201 (2017); IEEE - Institute of Electrical and Electronics Engineers (13\%) com fator de impacto 3,557 (2017); IFAC-Papersonline (8\%) 
com fator de impacto 0,260 (2017); Journal of Manufacturing Systems (8\%) com fator de impacto 1,548 (2017); Computers in Industry (7\%) com fator de impacto 1,028 (2017).

Fator de impacto é um indicador que reflete o número médio de citações de determinado artigo científico. Criado originalmente pelo Institute for Scientific Information é publicado anualmente observando-se a média das citações nos dois anos anteriores relacionada ao número de documentos pulicados neste mesmo período (Reuters, 2018). A equação 1 (anexo IV) demonstra matematicamente exemplo desta relação para um periódico "x" qualquer em 2010 (Moed \& Van Leeuwen, 1995).

O figura 1 (anexo II) apresenta evolução das publicações sendo possível observar o crescimento do interesse sobre o tema desta pesquisa no período 2015-2018, com variação média anual positiva de $284 \%$.

Considerando o nome do autor que inicia a lista de publicação, a figura 2 (anexo II) apresenta o país de origem das instituições de ensino destes autores, destacando a concentração de $60 \%$ do total pesquisado em: USA (19\%); China (13\%); Alemanha (9\%); Itália (8\%); Portugal (4\%), Espanha (4\%) e Brasil (4\%).

As publicações de autores brasileiros estão vinculadas às instituições de pesquisa: Pontifícia Universidade Católica do Paraná (5), Universidade de São Paulo (2), Universidade Tecnológica Federal do Paraná (1) e IBM Research - Brazil (1).

Após analisar os metadados, são encontradas 1.327 palavras-chave, sendo as mais recorrentes (86\%) apresentadas no quadro 1 (anexo III) em conjunto com o contexto desenvolvido nos artigos.

Para a ajudar na visualização gráfica das palavras-chaves, a figura 3 (anexo II) apresenta os clusters com o mínimo de 5 repetições, resultando em 17 palavras que atendem ao filtro aplicado. Evidência para CPS (cyber physical systems), decision making, industry 4.0 e loT (internet of things).

\subsubsection{Análise das citações}

(Neely, 2005) destaca a importância da frequência das citações de trabalhos individuais para identificar autores e trabalhos mais influentes no desenvolvimento da área de pesquisa. Este trabalho realiza análise de citações com base nos 245 artigos amostrais.

Em função da quantidade elevada de artigos, é aplicado um filtro considerando trabalhos com mais de 35 citações, resultando em 20 artigos. Com base nestes vinte artigos, é desenvolvida uma análise que relaciona as citações do artigo e o fator de impacto do periódico onde é publicado, originando um novo indicador - fator de impacto corrigido da citação (Lopes \& De Carvalho, 2012). A equação 2 (anexo IV) apresenta a relação destas variáveis.

São identificadas 3.045 citações, sendo 2.430 referentes aos 20 artigos mais citados, representando aproximadamente $80 \%$ do total. O quadro 2 (anexo III) apresenta a relação dos trabalhos mais citados (com mais de 35 citações), o fator de impacto corrigido, bem como, para evidenciar a 
influência do fator de impacto corrigido, é apresentada a variação da correção relativa a posição do fator de impacto original.

A figura 4 (anexo II) apresenta a evolução histórica destes vinte artigos. Nota-se que entre 2010 e 2014 há uma evolução das citações com variação média crescente de 17 artigos/ano. Contudo entre 2014 e 2018 esta média sobre para 236 artigos/ano, indicando crescimento do interesse acadêmico, atualidade do tema relativa ao período pesquisado e representatividade dos artigos citados.

Para reverenciar aos autores dos 20 artigos mais citados, a figura 5 (anexo II) apresenta os nomes e a quantidade de citações. A classificação é relativa ao fator de impacto corrigido da citação. Para fundamentar o resultado da pesquisa (análise de conteúdo) foi realizada a leitura completa destes 20 artigos na busca pela identificação de mecanismos que auxiliam a tomada de decisão em sistemas CPS.

\subsubsection{Análise das referências}

Em acréscimo aos artigos mais citados, foram analisadas as referências que contribuíram para a composição da fundamentação teórica dos trabalhos escolhidos (Lopes \& De Carvalho, 2012). Em função da representatividade de $87 \%$ na quantidade de artigos na amostra, foram utilizadas as bases SciencDirect e Scopus para realizar a análise dos autores mais referenciados. A figura 6 (anexo II) apresenta os clusters dos autores referenciados com número mínimo de 2 artigos por autor.

Com o objetivo de relacionar o tema desta pesquisa (tomada de decisão em sistemas ciberfísicos) com as referências, foram analisados os assuntos principais dos artigos referenciados. Dentre os temas pesquisados pelo cluster a esquerda da figura 6 (anexo II), composto pelos autores Liu, Y., Zhang, H., Leng, J., Chen, X, e Li, X., temos smart manufacturing, virtual models, stream mapping e digitalization of manufacturing. A direita da figura 6 (anexo II), no cluster composto pelos autores Dey, S. Quevedo, D. E., Ding, K., Shi, L. e Li, Y., os principais temas pesquisados foram cyber physical systems (CPS), decision making, information system e manufacturing.

\section{Resultado da pesquisa}

Anualmente, destacam-se a partir dos artigos mais citados (os 20 artigos) as contribuições para a tomada de decisão em sistemas ciber-físicos, onde em 2011 (Ilic, Xie, Khan, \& Moura, 2010) com 60\% das citações, informam a necessidade de considerar as informações históricas na configuração dos controladores automáticos durante o desenvolvimento de um modelo para tomada de decisão em sistemas CPS.

Em 2012, com 40\% das citações, (Swain, Couzin, \& Leonard, 2012) evidenciam a necessidade de introdução de intrumentos, a exemplo de câmeras bluetooth, que captem informações em tempo real para tomada de decisão em sistemas ciber-físicos. 
Entre 2013 e 2014 mais dois artigos foram destaques nas citações (34\%). (Tham \& Luo, 2013) desenvolveram sistema ciber-físico baseado na necessidade integração da cadeia produtiva para aumentar a acuracidade da previsão da demanda, e assim, melhorar a tomada de decisão. (Zhao, Suryanarayanan, \& Simoes, 2013) desenvolveram um sistema CPS que considera o feedback entre os agentes intervenientes para definir a tomada de decisão da carga de energia adequada em um processo produtivo.

Em 2015, o artigo de (Wang, Torngren, \& Onori, 2015) participou dos mais citados (8\%) apresentando avanços na tomada de decisão em sistemas ciber-físicos na manufatura, delineados pela conectividade e pela necessidade de controladores/atuadores descentralizados (sistemas computacionais com algoritmos inteligentes e depositados na nuvem).

Em 2016 mais dois artigos foram destaques entre os citados (15\%). (Leitão, Colombo, \& Karnouskos, 2016) apresentam possíveis dificuldades de gerenciamento (impacto da inoperância individual de módulos e necessidade de suporte na prestação de serviço em tempo real) e de sistemas de informação (integração de softwares heterogêneos e adaptação para uma gestão orientada pelo conhecimento) para administrar a tomada de decisão em sistemas ciber-físico. (Kang, et al., 2016) sugerem atenção especial a engenharia e aos fatores tecnológicos de comunicação envolvidos na tomada de decisão, devido ao alto nível de confiança requisitado pela produção inteligente.

Em 2017 outros dois artigos foram mais citados (13\%) onde (Babiceanu \& Seker, 2016) introduzem os conceitos de virtualização da produção, internet of things, big data para suportar a decisão autônoma e (Oesterreich \& Teuteberg, 2016) pesquisaram a automação da indústria 4.0 e suas principais tecnologias aplicadas na tomada de decisão.

De acordo com (Frazzon, Albrecht, Pires, \& Israel, 2018), o mais citado (11\%) dentre os artigos disponíveis até o momento da coleta de dados em 2018, sistemas ciber-físicos (CPS) necessitam de informações em tempo real para proporcionar uma tomada de decisão autônoma entre processos de produção e a cadeias de suprimentos, e assim direcionar as intenvenções humanas ao nível estratégico.

Em vista ao exposto, os sistemas ciber-físicos têm nível elevado de dependência dos processos de tomada de decisão, sobretudo por especificar esta decisão em carácter autônomo, que requisita eliminar os aspectos intuitivos.

Desta maneira, para assistir os objetivos da Indústria 4.0, os sistemas ciber-físicos devem tratar a tomada de decisão de maneira estratégica, considerando analisar as informações em tempo real.

Para isso, deve-se estender a integração sistêmica aos elementos da cadeia produtiva mais remotamente possível e promover a atualização das informações por intemédio controladores/decisores descentralizados que forneçam feedback do status operacional.

Portanto, a obtenção das informações de fontes variadas, em etapas produtivas diferentes e em momentos simultâneos dentro da cadeia produtiva fornecem uma visão preditiva aos sistemas CPS 
que auxiliam na sustentação de sua autonomia, e desta forma, produzem capacidade de resposta (antevisão) aos possíveis desvios que são projetados com base no desempenho dos processos anteriores.

\section{Conclusão}

De maneira geral, os artigos estudados fornecem resposta ao tema de pesquisa idealizando a tomada de decisão no ambiente ciber-físico sendo executada por controladores decentralizados.

Para descentralizar a decisão, ou seja, fornecer autonomia, é necessário promover integração sistêmica dos diversos atores intervenientes da cadeia produtiva que produz, por intermédio da análise de dados em tempo real (feedback operacional), atualização das informações históricas.

A informação sobre a disponibilidade ou não dos recursos planejados permite ao sistema CPS a correção na tomada de decisão no processo produtivo sobre qual o caminho deve ser adotado para compor o produto final com maior harmonia sob o prima do custo de produção.

Esta robusta rede integrada de informações eleva o nível de confiança na tomada de decisão para previsão de demanda e auxilia a manufatura a vencer o desafio de aumentar a variedade de produtos (customização em massa), que é um dos requisitos derivados da característica de consumo contemporânea ao período da pesquisa.

\section{Referências}

Babiceanu, R. F., \& Seker, R. (2016). Big Data and virtualization for manufacturing cyber-physical systems: A survey of the current status and future outlook. Computers in Industry, v. 81, pp. 128-137.

Bai, T. (2009). Strategic capabilities: antece dents to mass customization. International Conference on Electronic Commerce and Business Intelligence, pp. 302-305.

Bauer, H., Schoonmann, A., \& Reinhart, G. (2017). Approach for model-based change impact analysis in factory systems. IEEE International Symposium on Systems Engineering.

Broadus, R. N. (1987). Toward a definition of "bibliometrics". Scientometrics, v. 12, pp. 373-379.

Camarinha-Matos, L. M., \& Afsarmanesh, H. (2014). Collaborative systems for smart environments: Trends and challenges. IFIP Advances in Information and Communication Technology, v. 434, pp. 3-15. 
De Matos, E. B., Niyama, J. K., Neto, L. M., \& Marques, M. M. (2012).

Congresso ANPCONT: análise bibliométrica descritiva e avaliativa dos artigos publicados de 2007 a 2011. Enfoque: Reflexão Contábil, v. 31, pp. 73-88.

Eck, N. J., \& Waltman, L. (2018). VOSviewer - visualizing scientific landscapes. Version 1.6.7 Copyright 2009-2018.

Frazzon, E. M., Albrecht, A., Pires, M., \& Israel, E. (2018). Hybrid approach for the integrated scheduling of production and transport processes along supply chains. International Journal of Production Research, v. 56, pp. 2019-2035.

Google LLC. (20 de Dez de 2018). Articles. Fonte: Google Scholar: https://scholar.google.com.br/

Gutiérrez-Salcedo, M., Martínez, M., Moral-Munoz, J., Herrera-Viedma, E., \& Cobo, M. (2018). Some bibliometric procedures for analyzing and evaluating research fields. Applied Intelligence, v. 48, pp. $1275-1287$.

Henriques, F., \& Miguel, P. A. (2015). Modularidade na indústria automotiva: seleção de um portfólio de artigos para pesquisa por meio de uma análise bibliométrica. Exacta-EP, v. 13, pp. 389401.

Ilic, M. D., Xie, L., Khan, U. A., \& Moura, J. M. (2010). Modeling of Future Cyber-Physical Energy Systems for Distributed Sensing and Control. IEEE Transactions on Systems, v. 40, pp. 825-838.

Kang, H. S., Lee, J. Y., Choi, S., Kim, H., Park, J. H., Son, J. Y., Noh, S. D. (2016). Smart manufacturing: Past research, present findings, and future directions. International Journal of Precision Engineering and Manufacturing, v. 3, pp. 111-128.

Karlsson, C. (2008). Researching operations management. Research methods for operational management, pp. 41-44.

Leitão, P., Colombo, A. W., \& Karnouskos, S. (2016). Industrial automation based on cyber-physical systems technologies: Prototype implementations and challenges. Computers in Industry, v. 81, pp. 11-25. 
Lopes, A. P., \& De Carvalho, M. M. (2012). Evolução da literatura de inovação em relações de cooperação: um estudo bibliométrico num período de vinte anos. Gestão e Produção, v. 19, pp. 203-217.

Mcburney, M. K., \& Novak, P. L. (2002). What is bibliometrics and why should you care? IEEE International Professional Communication Conference, pp. 108-114.

Microsoft. (2016). Excel ${ }^{\circledast}$ MSO (16.0.4266.1001). Microsoft Corporation. All Rights Reserved.

Moed, H. F., \& Van Leeuwen, T. N. (1995). Improving the accuracy of institute for scientific information's journal impact factors. Journal of the American Society for Information Science, v. 46, pp. $461-467$.

Neely, A. (2005). The evolution of performance measurement research: developments in the last decade and a research agenda for the next. International Journal of Operations \& Production Management, v. 25, pp. 1264-1277.

Oesterreich, T. D., \& Teuteberg, F. (2016). Understanding the implications of digitisation and automation in the context of Industry 4.0: A triangulation approach and elements of a research agenda for the construction industry. Computers in Industry, v. 83, pp. 121-139.

Osorio, N. L., \& Otieno, A. W. (2008). A survey of manufacturing engineering databases. Collection Building, v. 27, pp. 22-29.

Papa, G., Zurutuza, U., \& Uribeetxeberria, R. (2016). Cyber physical system based proactive collaborative maintenance. Proceedings of 2016 International Conference on Smart Systems and Technologies, pp. 173-178.

Pereira, R. A., Ribeiro, M. S., \& Bianchini, D. (2014). Tomada de decisão mediante aos impactos da turbulência nas convergências tecnológicas no mercado de telefonias móveis: Um estudo à luz da estabilidade dinâmica. Exacta - EP, v. 12, pp. 105-122.

Prasad, S., \& Tata, J. (2005). Publication patterns concerning the role of teams/groups in the information systems literature from 1990 to 1999. Information and Management, v. 42, pp. 1137-1148. 
Reuters, T. (12 de mai de 2018). Company history (historical highlights

from across Thomson Reuters). Fonte: https://www.thomsonreuters.com/en/aboutus/company-history.html

Swain, D. T., Couzin, I. D., \& Leonard, N. E. (2012). Real-Time Feedback-Controlled Robotic Fish for Behavioral Experiments With Fish Schools. Proceedings of the IEEE, v. 100.

Tham, C. K., \& Luo, T. (2013). Sensing-Driven Energy Purchasing in Smart Grid Cyber-Physical System. IEEE Transactions on Systems, v. 43.

Vaidya, S., Ambad, P., \& Bhosle, S. (2018). Industry 4.0 - A Glimpse. Procedia Manufacturing - Elsevier, v. 20 , pp. $233-238$.

Wang, L., Torngren, M., \& Onori, M. (2015). Current status and advancement of cyber-physical systems in manufacturing. Journal of Manufacturing Systems, v. 37, pp. 517-527.

Wang, Q., \& Sun, X. (2018). The international journal of production research in the past, the present and the future: a bibliometric analysis. International Journal of Production Research.

Williams, R., \& Bornmann, L. (2016). Sampling issues in bibliometric analysis. Journal of Informetrics, v. 10, pp. $1225-1232$.

Zhao, P., Suryanarayanan, S., \& Simoes, M. G. (2013). An Energy Management System for Building Structures Using a Multi-Agent Decision-Making Control Methodology. IEEE Transactions on Industry Applications, v. 49, pp. 322-330. 
Tabela 1

Publicações por periódico e por ano

\begin{tabular}{|c|c|c|c|c|c|c|c|c|c|c|c|}
\hline \multirow{2}{*}{ Periódicos } & \multicolumn{9}{|c|}{ Ano (2010 - 2018) } & \multirow{2}{*}{ Total } & \multirow{2}{*}{$\%$} \\
\hline & 10 & 11 & 12 & 13 & 14 & 15 & 16 & 17 & 18 & & \\
\hline Procedia Manufacturing & & & & & & 1 & 2 & 41 & 21 & 65 & $27 \%$ \\
\hline IEEE - Inst. of Elect. and Elec. Eng. & 1 & & 1 & 2 & & 3 & 6 & 7 & 12 & 32 & $13 \%$ \\
\hline IFAC-Papersonline & & & & & & 2 & 5 & 2 & 10 & 19 & $8 \%$ \\
\hline Journal of Manufacturing Systems & & & & & & 3 & 1 & 7 & 8 & 19 & $8 \%$ \\
\hline Computers in Industry & & & & & & & 8 & 4 & 4 & 16 & $7 \%$ \\
\hline Procedia Engineering & & 1 & & & & 1 & & 4 & & 6 & $2 \%$ \\
\hline ZWF / Int. J. of Adv. Manuf. Techn. & & & & & & & $2 / 0$ & & $0 / 3$ & 5 & $2 \%$ \\
\hline Sensors / Journal Int. Meas. Confed. & & & & & & & & $2 / 0$ & $0 / 2$ & 4 & $2 \%$ \\
\hline Comp.Ind.Eng./Proc.Saf. Envi. Prot. & & & & & & & & $1 / 0$ & $4 / 2$ & 7 & $3 \%$ \\
\hline ICIC Express Letters / Energies & & & & & $1 / 0$ & & & & $0 / 2$ & 3 & $1 \%$ \\
\hline Concurrent Eng. Research and Appl. & & & & & & 1 & & 1 & & 2 & $1 \%$ \\
\hline Cybernetics And Systems & & & & & & 1 & 1 & & & 2 & $1 \%$ \\
\hline Expert Systems with Applications & & & & & & & 1 & & 1 & 2 & $1 \%$ \\
\hline Int. Journal of Production Research & & & & & & & & 2 & 2 & 4 & $2 \%$ \\
\hline Journal of The Chinese Inst. of Eng. & & & & & & & & 2 & & 2 & $1 \%$ \\
\hline Productivity Management & & & & & & & 1 & 1 & & 2 & $1 \%$ \\
\hline DYNA / Engineering & & & & & & & & $1 / 1$ & & 2 & $1 \%$ \\
\hline Machines / Production Engineering & & & & & & & & $1 / 1$ & & 2 & $1 \%$ \\
\hline Risk Anal. / Int. J.Dist. Sensor Net & & & $1 / 1$ & & & & & & & 2 & $1 \%$ \\
\hline Assembly /ATutomatisierungstechnik & & & & & & & $1 / 1$ & & & 2 & $1 \%$ \\
\hline Manuf. Letters / Int.Prec.Eng.Manuf. & & & & & & & $1 / 1$ & & & 2 & $1 \%$ \\
\hline ACM Trans.Aut.Adapt.Syst. / CEAS & & & & $1 / 0$ & & & & & $0 / 1$ & 2 & $1 \%$ \\
\hline Advances In Mech. Eng./Energy & & & & & & & & & $1 / 1$ & 2 & $1 \%$ \\
\hline Cont. Eng.Practice / J. Infr. Systems & & & & & & $1 / 0$ & & & $0 / 1$ & 2 & $1 \%$ \\
\hline Auto. Ele.Po Sys./J.Signal Proc. Sys. & & & & & & & & $1 / 0$ & $0 / 1$ & 2 & $1 \%$ \\
\hline Int. J. Aut.Techn./Xitong Fangzhen & & & & & & $1 / 0$ & & & $0 / 1$ & 2 & $1 \%$ \\
\hline Int. J. Critical Infra./Info. Scien. & & & & & & & & $1 / 0$ & $0 / 1$ & 2 & $1 \%$ \\
\hline Int. J. Prod. Econo. / GEOMATE & & & & & & & & $1 / 0$ & $0 / 1$ & 2 & $1 \%$ \\
\hline Journal Eng. Design / Geomechanik & & & & & & & & $1 / 0$ & $0 / 1$ & 2 & $1 \%$ \\
\hline J.Ind. Infor. Integr. / J. Mech. Eng. & & & & & & $0 / 1$ & & $1 / 0$ & 1 & 3 & $1 \%$ \\
\hline Journal Mach.Eng. / J.Spa. Saf. Eng. & & & & & & & & & $1 / 1$ & 2 & $1 \%$ \\
\hline Mng. Prod. Eng. Rev. /Rapid Prot. & & & & & & $1 / 0$ & & & $0 / 1$ & 2 & $1 \%$ \\
\hline Applied Intell. / Dianli Zidonghua & & & & $1 / 0$ & & & & & $0 / 1$ & 2 & $1 \%$ \\
\hline Chinese Journal of Mech. Eng. & & & & & & & & 1 & & 1 & $0,4 \%$ \\
\hline Journal of Dalian Univ. of Technol. & & & & & 1 & & & & & 1 & $0,4 \%$ \\
\hline Adv. Eng. Informatics & & & & & & & 1 & & & 1 & $0,4 \%$ \\
\hline Found. Trends in Systems Control & & & & & & 1 & & & & 1 & $0,4 \%$ \\
\hline Int. Journal Comp. Integrat. Manu. & & & & & & & 1 & & & 1 & $0,4 \%$ \\
\hline Journal Ambient Intell.Smart Envir. & & & & & & & & 1 & & 1 & $0,4 \%$ \\
\hline Journal Comp. and Info. Sc. In Eng. & & & & & & & & 1 & & 1 & $0,4 \%$ \\
\hline Journal of Comp. Science And Eng. & & & & & & 1 & & & & 1 & $0,4 \%$ \\
\hline Management Sc. and Eng. Dept. & & & & & & & & 1 & & 1 & $0,4 \%$ \\
\hline Management Systems in Prod. Eng. & & & & & & & & 1 & & 1 & $0,4 \%$ \\
\hline Mechanical SystemsSignal Proc. & & & & 1 & & & & & & 1 & $0,4 \%$ \\
\hline Microelectronic Engineering & & & & & & 1 & & & & 1 & $0,4 \%$ \\
\hline Smt Surface Mount Tech. Magazine & & & & & & 1 & & & & 1 & $0,4 \%$ \\
\hline Outros & & & & & & & & & 7 & 7 & $3 \%$ \\
\hline Total & 1 & 1 & 3 & 5 & 2 & 20 & 33 & 88 & 92 & 245 & - \\
\hline
\end{tabular}

Fonte: Autores. 
Figura 1

Histórico de publicações

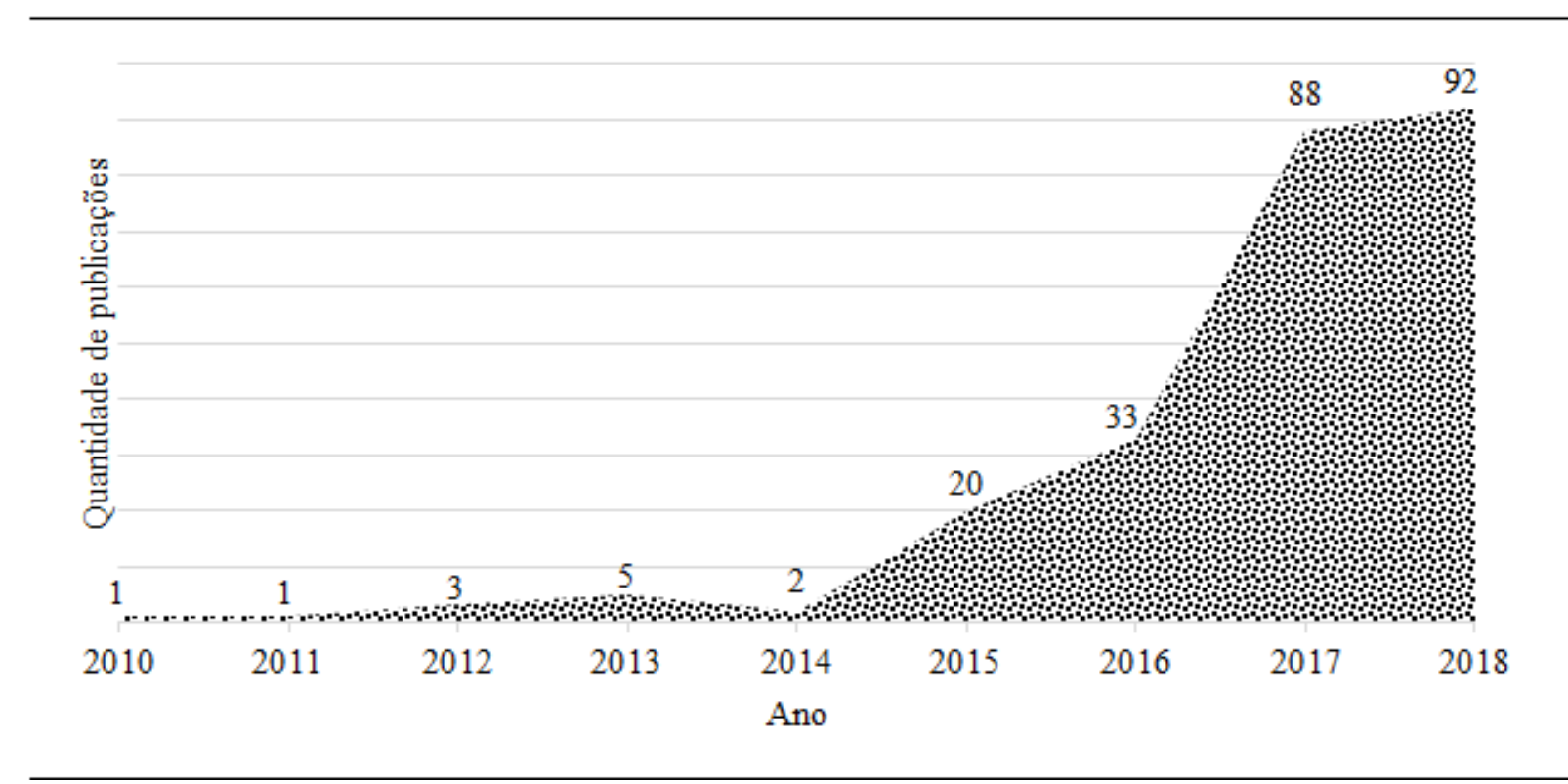

Fonte: Autores.

Figura 2

Quantidade de artigo por país
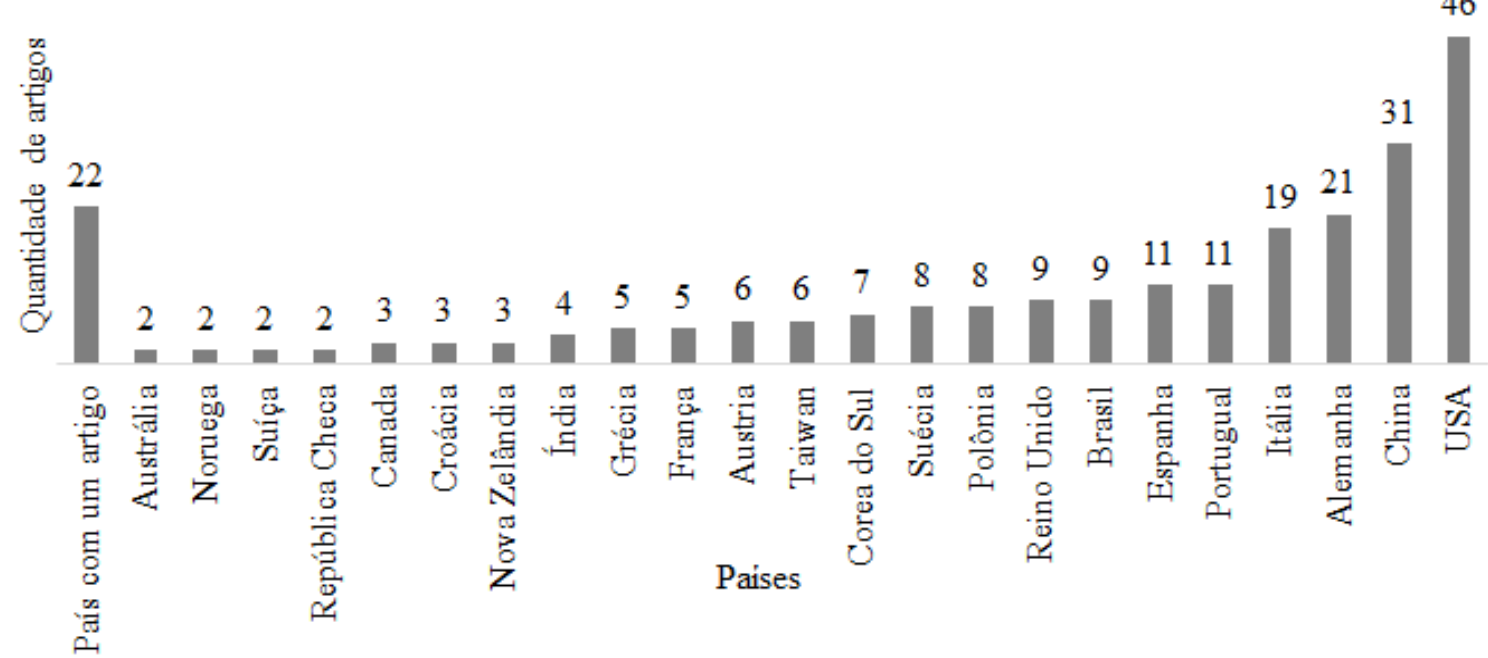

Fonte: Autores. 
Figura 3

Clusters das palavras chaves com o mínimo de 5 repetições

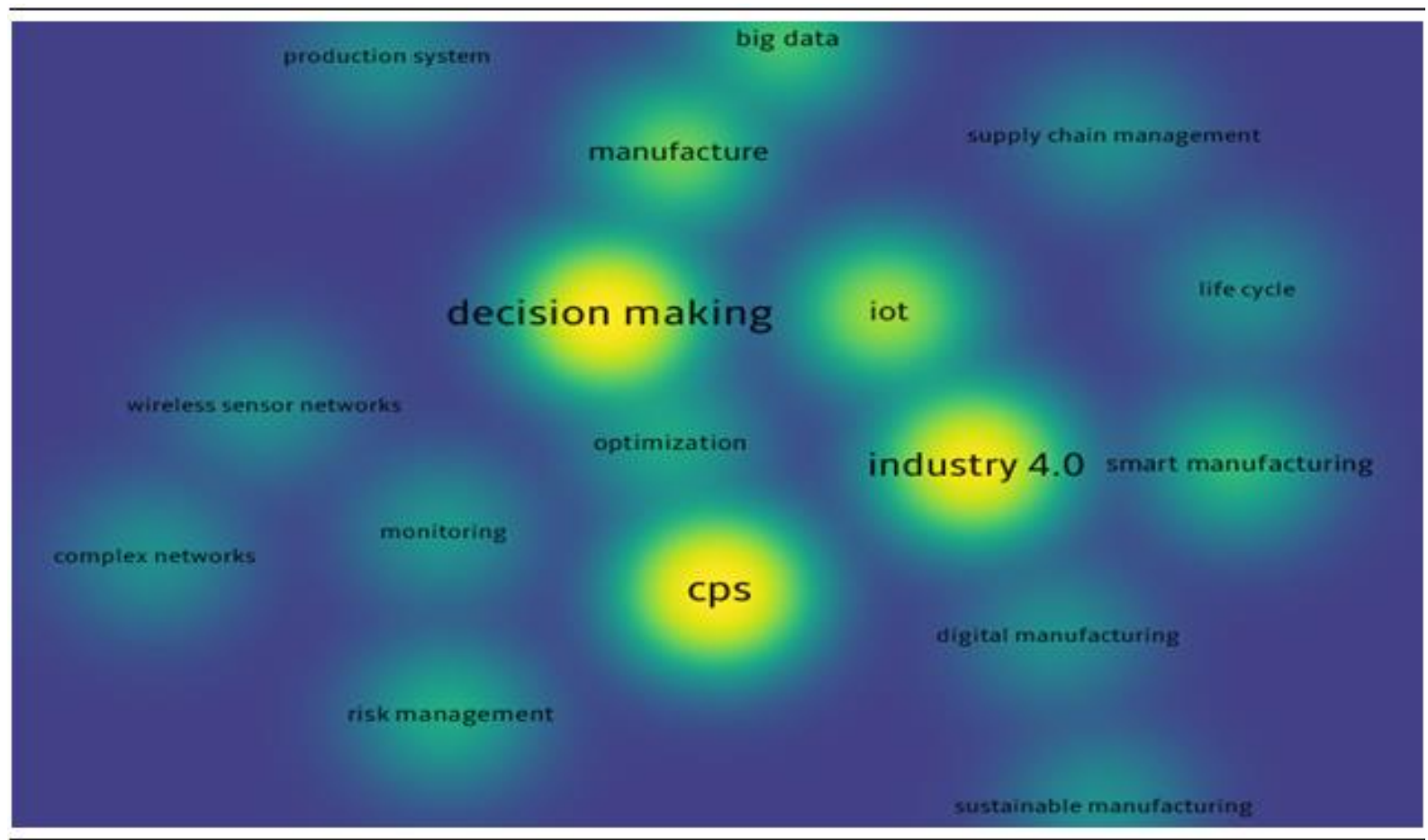

Fonte: (Eck \& Waltman, 2018; autores).

Figura 4

Evolução histórica das citações dos 20 artigos com mais representativos

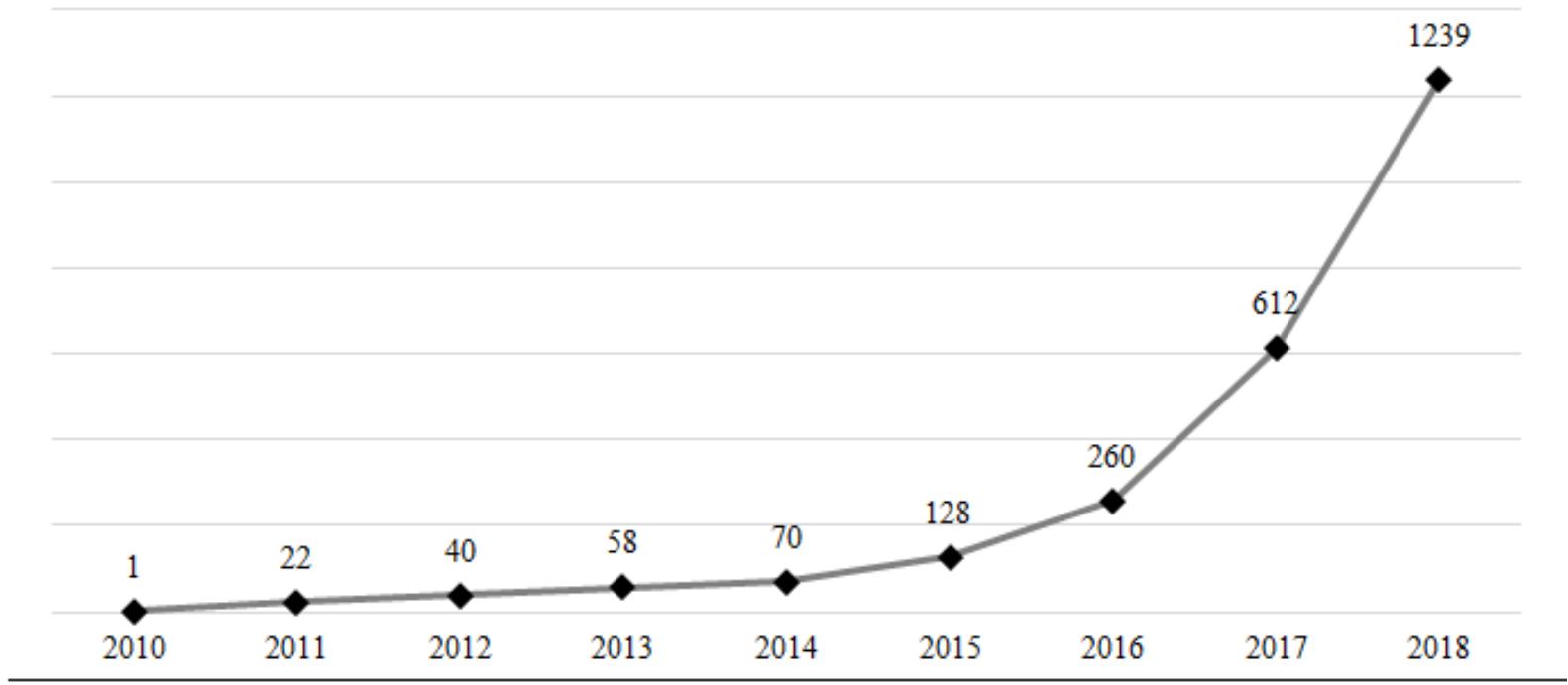

Fonte: Autores. 
Santana, H. L. \& Assumpção, M. R. P. (2023, jan./mar.). Tomada de decisão em sistemas ciberfísicos: uma análise bibliométrica

Figura 5

Nomes dos autores e citações recebidas por ano

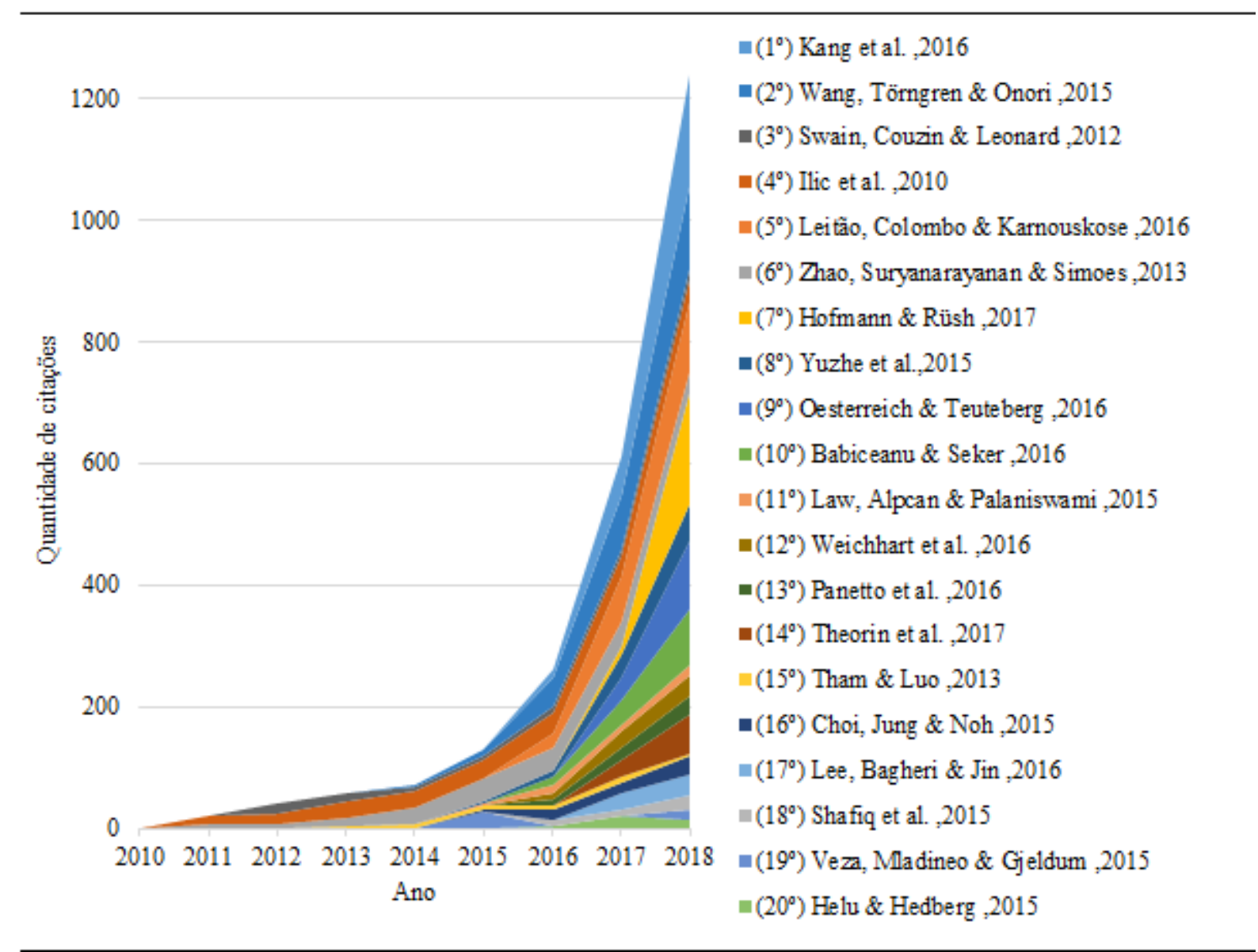

Fonte: Autores. 
Figura 6

Clusters de autores referenciados com mínimo de 2 artigos publicados

liu, y.

zhang, h.

leng, j.

ding, $\mathrm{k}$.

chen, $x$.

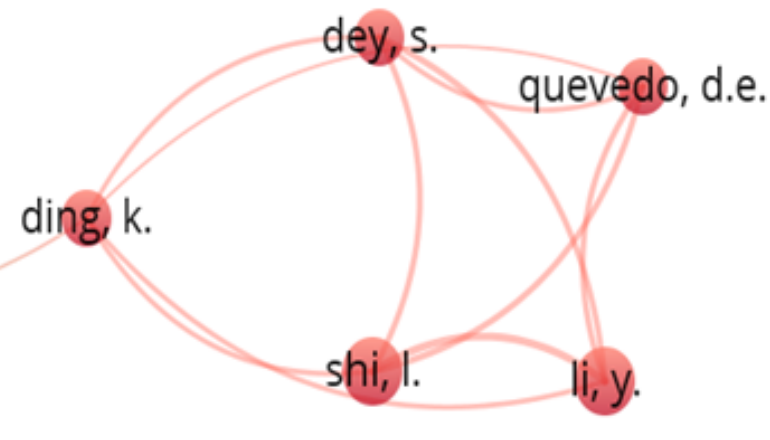

li, $x$.

Fonte: (Eck \& Waltman, 2018; autores). 


\section{Anexo III - Quadros}

\section{Quadro 1}

Palavras chaves mais representativas e a descrição do contexto nos artigos

\begin{tabular}{|c|c|c|c|c|}
\hline Ordem & Palavras-chaves & Contexto desenvolvido nos artigos & Qtd & \% participação \\
\hline 1 & $\begin{array}{l}\text { Sistema ciber } \\
\text { físico (CPS) }\end{array}$ & $\begin{array}{l}\text { Foi apontando nos artigos a demanda pelo } \\
\text { desenvolvimento de sistemas com decisão } \\
\text { colaborativa entre equipamentos para o } \\
\text { roteamento da produção. }\end{array}$ & 279 & $21 \%$ \\
\hline 2 & $\begin{array}{l}\text { Tomada de } \\
\text { decisão }\end{array}$ & $\begin{array}{l}\text { Esteve presente nos artigos devido as diversas } \\
\text { variáveis oriundas do ambiente produtivo a serem } \\
\text { consideradas na tomada de decisão autônoma. }\end{array}$ & 265 & $20 \%$ \\
\hline 3 & Indústria 4.0 & $\begin{array}{l}\text { Foi destaque nos artigos onde o sistema de } \\
\text { produção contempla digitalização, automação dos } \\
\text { processos por meio de sensoriamento, troca de } \\
\text { dados e loT. }\end{array}$ & 239 & $18 \%$ \\
\hline 4 & $\begin{array}{l}\text { Internet das } \\
\text { coisas (IoT) }\end{array}$ & $\begin{array}{l}\text { Apareceu nos artigos indicando a relação dos } \\
\text { equipamentos com a internet, e posterior relação } \\
\text { entre entre si para gerenciamento dos gargalos. }\end{array}$ & 119 & $9 \%$ \\
\hline 5 & Produção & $\begin{array}{l}\text { Foi visualizado nos artigos a tendência dos novos } \\
\text { sistemas de produção serem colaborativos e não } \\
\text { mais competitivos, gerando uma relação ganha- } \\
\text { ganha. }\end{array}$ & 80 & $6 \%$ \\
\hline 6 & Big data & $\begin{array}{l}\text { Foi ressaltado nos artigos a necessidade de } \\
\text { sistemas que transformem o volume de dados } \\
\text { disponível em informações com objetividade e } \\
\text { flexibilidade. }\end{array}$ & 66 & $5 \%$ \\
\hline 7 & $\begin{array}{l}\text { Produção } \\
\text { inteligente }\end{array}$ & $\begin{array}{l}\text { Foi frequente nos artigos a intenção de tratar } \\
\text { ambientes com sensonriamento para integração e } \\
\text { controle computacional e decisões autônomas. }\end{array}$ & 53 & $4 \%$ \\
\hline 8 & $\begin{array}{l}\text { Gerenciamento } \\
\text { de risco }\end{array}$ & $\begin{array}{l}\text { Foi evidenciado nos artigos o crescimento da } \\
\text { complexidade dos riscos envolvidos na tomada de } \\
\text { decisão autônoma e a relação humana. }\end{array}$ & 40 & $3 \%$ \\
\hline \multicolumn{3}{|c|}{ Total desta seleção } & 1.141 & $86 \%$ \\
\hline \multicolumn{3}{|c|}{ Total geral } & 1.327 & \\
\hline
\end{tabular}

Fonte: Autores. 
Quadro 2

Artigos com mais de 35 citações (ordem decrescente do fator de impacto corrigido)

\begin{tabular}{|c|c|c|c|c|c|}
\hline $\mathrm{Nr}$ & Breve descrição dos artigos & Citações & $\begin{array}{l}\text { Fator de } \\
\text { Impacto } \\
\text { original }\end{array}$ & $\begin{array}{l}\text { Fator de } \\
\text { impacto } \\
\text { corrigido }\end{array}$ & $\begin{array}{l}\text { Variação } \\
\text { da } \\
\text { correção }\end{array}$ \\
\hline 1 은 & $\begin{array}{l}\text { Smart manufacturing: Past research, present } \\
\text { findings, and future directions }\end{array}$ & 259 & 3,494 & 1164 & $\begin{array}{l}\text { Subiu } 3 \\
\text { posições }\end{array}$ \\
\hline 20 & $\begin{array}{l}\text { Current status and advancement of cyber-physical } \\
\text { systems in manufacturing }\end{array}$ & 283 & 2,770 & 1067 & $\begin{array}{l}\text { Subiu } 6 \\
\text { posições }\end{array}$ \\
\hline 30 & $\begin{array}{l}\text { Real-time feedback-controlled robotic fish for } \\
\text { behavioral experiments with fish schools }\end{array}$ & 86 & 9,237 & 880 & $\begin{array}{l}\text { Desceu } 2 \\
\text { posições }\end{array}$ \\
\hline 40 & $\begin{array}{l}\text { Modeling of Future Cyber-Physical Energy Systems } \\
\text { for Distributed Sensing and Control }\end{array}$ & 217 & 2,937 & 854 & $\begin{array}{l}\text { Subiu } 1 \\
\text { posição }\end{array}$ \\
\hline 50 & $\begin{array}{l}\text { Industrial automation based on cyber-physical } \\
\text { systems technologies: Prototype implementations } \\
\text { and challenges }\end{array}$ & 217 & 2,691 & 801 & $\begin{array}{l}\text { Subiu } 4 \\
\text { posições }\end{array}$ \\
\hline 60 & $\begin{array}{l}\text { An energy management system for building } \\
\text { structures using a multi-agent decision-making } \\
\text { control methodology }\end{array}$ & 201 & 2,937 & 791 & Estável \\
\hline 70 & $\begin{array}{l}\text { Industry } 4.0 \text { and the current status as well as future } \\
\text { prospects on logistics }\end{array}$ & 200 & 2,691 & 738 & $\begin{array}{l}\text { Subiu } 6 \\
\text { posições }\end{array}$ \\
\hline 80 & $\begin{array}{l}\text { Jamming attacks on remote state estimation in } \\
\text { cyber-physical systems: A game-theoretic approach }\end{array}$ & 111 & 4,270 & 585 & $\begin{array}{l}\text { Desceu } 5 \\
\text { posições }\end{array}$ \\
\hline 9 은 & $\begin{array}{l}\text { Understanding the implications of digitisation and } \\
\text { automation in the context of Industry 4.0: A } \\
\text { triangulation approach and elements of a research } \\
\text { agenda for the construction industry }\end{array}$ & 151 & 2,691 & 557 & $\begin{array}{l}\text { Subiu } 2 \\
\text { posições }\end{array}$ \\
\hline 10 은 & $\begin{array}{l}\text { Big Data and virtualization for manufacturing cyber- } \\
\text { physical systems: A survey of the current status and } \\
\text { future outlook }\end{array}$ & 143 & 2,691 & 528 & Estável \\
\hline 11 은 & $\begin{array}{l}\text { Security games for risk minimization in automatic } \\
\text { generation control }\end{array}$ & 47 & 5,680 & 314 & $\begin{array}{l}\text { Desceu } 9 \\
\text { posições }\end{array}$ \\
\hline 120 & $\begin{array}{l}\text { Challenges and current developments for Sensing, } \\
\text { Smart and Sustainable Enterprise Systems }\end{array}$ & 68 & 2,691 & 251 & Estável \\
\hline 13 운 & $\begin{array}{l}\text { New perspectives for the future interoperable } \\
\text { enterprise systems }\end{array}$ & 64 & 2,691 & 236 & $\begin{array}{l}\text { Subiu } 1 \\
\text { posição }\end{array}$ \\
\hline 140 & $\begin{array}{l}\text { An event-driven manufacturing information system } \\
\text { architecture for Industry } 4.0\end{array}$ & 88 & 1,463 & 217 & $\begin{array}{l}\text { Subiu } 1 \\
\text { posição }\end{array}$ \\
\hline
\end{tabular}




\begin{tabular}{|c|c|c|c|c|c|}
\hline $\mathrm{Nr}$ & Breve descrição dos artigos & Citações & $\begin{array}{l}\text { Fator de } \\
\text { Impacto } \\
\text { original }\end{array}$ & $\begin{array}{l}\text { Fator de } \\
\text { impacto } \\
\text { corrigido }\end{array}$ & $\begin{array}{l}\text { Variação } \\
\text { da } \\
\text { correção }\end{array}$ \\
\hline 150 & $\begin{array}{l}\text { Sensing-driven energy purchasing in smart grid } \\
\text { cyber-physical system }\end{array}$ & 42 & 2,937 & 165 & $\begin{array}{l}\text { Desceu } 8 \\
\text { posições }\end{array}$ \\
\hline 160 & $\begin{array}{l}\text { Virtual reality applications in manufacturing } \\
\text { industries: Past research, present findings, and } \\
\text { future directions }\end{array}$ & 67 & 1,000 & 134 & Estável \\
\hline 170 & Introduction to cyber manufacturing & 59 & 0,981 & 117 & Estável \\
\hline 18 은 & $\begin{array}{l}\text { Virtual Engineering Object (VEO): Toward } \\
\text { Experience-Based Design and Manufacturing for } \\
\text { Industry } 4.0\end{array}$ & 47 & 0,930 & 91 & Estável \\
\hline 190 & $\begin{array}{l}\text { Managing Innovative Production Network of Smart } \\
\text { Factories }\end{array}$ & 42 & 0,263 & 53 & Estável \\
\hline 200 & $\begin{array}{l}\text { Enabling Smart Manufacturing Research and } \\
\text { Development using a Product Lifecycle Test Bed }\end{array}$ & 38 & 0,105 & 42 & Estável \\
\hline
\end{tabular}

Fonte: Autores.

\section{Anexo IV - Equações}

\section{Equação 1}

Modelo matemático para fator de impacto. Fonte: (Moed \& Van Leeuwen, 1995)

$$
\begin{aligned}
& \text { Fator de impacto de " } x "(\text { ano 2010) }= \\
& =\frac{\sum \text { citações do periódico " } x " \text { publicadas em } 2008 \text { e } 2009}{\sum \text { artigos publicados em } 2008 \text { e } 2009}
\end{aligned}
$$

\section{Equação 2}

Modelo matemático para corrigir o número de citação. Fonte: (Lopes \& De Carvalho, 2012)

$$
\begin{gathered}
\text { Índice corrigido de citação }= \\
=[\text { número de citações do artigo * (fator de impacto }+1)]
\end{gathered}
$$

